\title{
Luminance thresholds for circular and bar-shape stimuli
}

\author{
STUART APPELLE \\ State University of New York, College at Brockport, Brockport, New York 14420
}

\begin{abstract}
Studies involving the contribution of stimulus form to the detection threshold for light are equivocal, but theoretical considerations suggest that circular stimuli should yield lower thresholds than bars. This hypothesis was tested by obtaining luminance thresholds for circular and bar-shaped targets presented from 5 to $60 \mathrm{deg}$ into the periphery. Thresholds for circles were significantly lower than thresholds for bars. The difference ranged from about .4 log units at 5 deg to .2 log units at 60 deg eccentricity.
\end{abstract}

Graham, Brown, and Mote (1939) determined thresholds for circular stimuli of different sizes. They concluded that the excitatory contribution of any particular area of a stimulus decreases with its distance from the center of that stimulus. Elaborating on this theme, some theorists (Blackwell, 1957; Kincaid, Blackwell, \& Kristofferson, 1960) predict that circular targets should yield lower luminance thresholds than any other forms. However, the experimental support for this theory is equivocal (Zusne, 1970).

To determine whether circular patches of light are, in fact, more visible than extended configurations, luminance thresholds were obtained for circular and bar-shaped stimuli presented from 5 to $60 \mathrm{deg}$ into the periphery. Since the total area or solid visual angle subtended by these test objects was equal, and since they were presented to the same general retinal loci, only the configurational differences in the stimuli could account for differences in required threshold energy. Thresholds were determined with binocular (free) viewing and at photopic levels of adaptation. These conditions were chosen to make the data more relevant to typical search conditions occurring outside the laboratory.

\section{METHOD}

\section{Subjects}

The subjects were 20 paid volunteers with normal uncorrected vision.

\section{Apparatus}

A central "grain-of-wheat" fixation bulb (set just above foveal threshold) and eight stimulus lamps positioned at 5, 20, 40 , and $60 \mathrm{deg}$ both left and right of the fixation point were mounted on a 180-deg perimeter at a viewing distance of $58 \mathrm{~cm}$. The light sources were Sylvania electroluminescent panels. This lighting system provides a luminance output which, over the ranges used, is proportional to applied voltage and has no accompanying change in spectral output. Additionally, brightness and spectral energy (peak occurring at $505 \mathrm{~nm}$ ) is uniform across the panel surface. These convenient sources have proven useful in similar applications of perimetry (Leibowitz \& Appelle, 1969; Abernethy \& Leibowitz, 1971).

This study was supported in part by State University of New York Grant 022-7168A and National Science Foundation Institutional Grant 22-A010 to the author. I thank David Burrows who sponsors this article and takes full editorial responsibility for it.
All lights were at the subjects' eye level. The stimulus lights were fitted with bar-shape apertures, $1.48 \mathrm{deg} \times 3 \mathrm{~min}$ of arc, or circular apertures equivalent in area $(18 \mathrm{~min}$ diam) to the bar shapes. A head and chin rest was provided to maintain proper fixation during the testing sessions. To maintain photopic conditions, subjects viewed a diffusing screen at a distance of $80 \mathrm{~cm}(72 \times 72 \mathrm{deg})$ illuminated by a $61.5 \mathrm{fc}$ source. Luminance of the adapting field was $1.2 \log \mathrm{fL}$. Detection of the test stimulus was indicated by operation of a switch directly below the base of the chin rest, which also activated the intertrial adaptation light. To eliminate extraneous cues, the adaptation light was extinguished during each trial, at which time the test chamber was completely dark.

\section{Procedure}

To begin each trial, the subject threw a switch which extinguished all lights in the experimental chamber, except for the fixation light and the peripheral light being presented. Some dark adaptation could be expected to take place during this period, but the effect would be the same for both groups and would not affect the relative differences between circles and lines. In addition, pilot data (confirmed by the recorded data) indicate no correlation between time in the dark (trial duration) and threshold luminance for these brief intervals involved (an average of $19 \mathrm{sec}$ ). That is, threshold was a function of stimulus eccentricity and configuration, not trial duration.

The subjects sat positioned in a head and chin rest facing the perimeter, and were instructed to maintain fixation at all times. At the commencement of each trial, a stimulus light was activated at 2-sec intervals for a duration of $100 \mathrm{msec}$. Between flashes, intensity was increased by increasing the driving voltage in regular intervals according to the ascending method of limits. Appropriate starting points for the series were determined in pilot data for each eccentricity. Subjects were asked to signal the moment of detection by saying "left" or "right" when the light appeared (indicating its visual field) and pulling the switch located above their lap. This terminated the trial, gave a record of trial duration, and reactivated the adaptation light in the chamber. Subjects viewed the adaptation field for 1-min intervals between trials. This interval was sufficient to maintain thresholds at stable photopic levels (again indicating that the trials themselves contributed little in the way of dark adaptation). However, sufficient practice trials always had to precede recording of data so that threshold levels could stabilize. This requirement was anticipated since significant reductions in binocularly obtained peripheral thresholds, after short practice periods, have been reported before (Leibowitz \& Appelle, 1969; Abernethy \& Leibowitz, 1971).

Two groups of subjects were tested, with 10 subjects randomly assigned to each group. One group was tested with the line stimuli and the other group with the circular targets. For the line group, stimuli were presented in vertical, horizontal, 45, and $135 \mathrm{deg}$ orientations, in a predetermined 


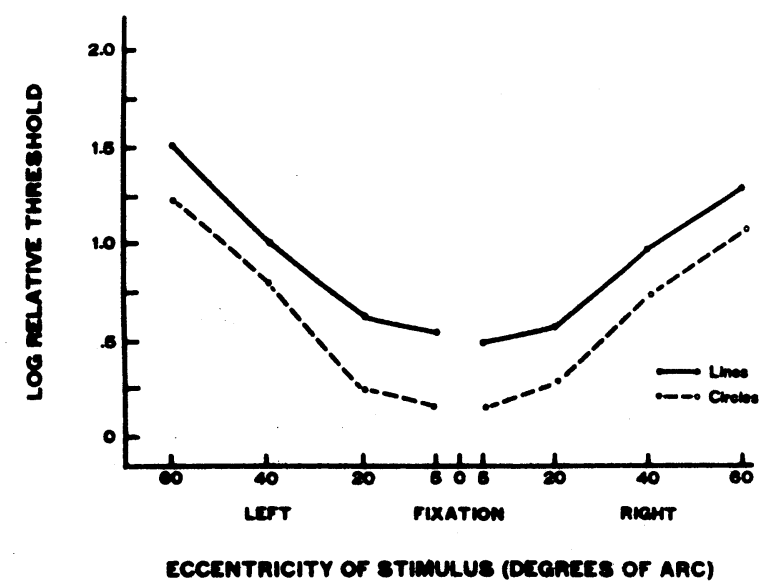

Figure 1. Log threshold luminance as a function of stimulus eccentricity and shape.

randomized order. Three threshold measurements were made for each orientation at each eccentricity. These 96 determinations were made over two $1-h$ testing sessions, usually 1 day apart. The subjects were not given any information about the orientation of the stimuli, nor were they asked to report on stimulus orientation. In fact, since only light detection was required, they were not able to do so. Form recognition requires $25 \%$ greater luminance than simple light detection (Helson \& Fehrer, 1932). Subjects were specifically questioned about stimulus appearance when testing was completed, and all subjects reported the stimuli as formless patches of light. Therefore, while these stimuli were physically different from the circular targets, they were equivalent subjectively.

Three threshold determinations were made at each eccentricity for the circular targets. Only one testing session, lasting about $1 \mathrm{~h}$, was needed to get these 24 measurements. For all stimuli, luminance values were first recorded in terms of applied voltage and later converted into relative units with a Photovolt multiplier photometer. Each light source was calibrated separately. The reference level for these relative values was determined in absolute terms with a Macbeth illuminometer.

\section{RESULTS}

In order to compare circles with elongated shapes per se, thresholds for the bar shapes have been averaged for the four orientations used. This procedure is justified, since thresholds for verticals and horizontals are not significantly different from each other, nor are left and right slanting obliques. And while oblique thresholds are lower than horizontals and verticals (Appelle, 1973), this difference is too small (.02 log units) to have contributed to the difference between lines and circles. These differences are presented in Figure 1 (zero on the ordinate corresponds to a luminance of $-2.25 \log \mathrm{fL}$ ). Thresholds for bars are consistently higher than those for circle targets. This difference is about $.4 \log$ units at $5 \mathrm{deg}$ and decreases to about $.2 \log$ units at $60 \mathrm{deg}$. Mean thresholds were compared in a three factor analysis of variance (Target Shape by Eccentricity by Visual Half Field; orientation was not included as a fartor since a representative measurement for bar shapes was desired, and since orientation was found to make no contribution statistically). The main effects of eccentricity $[\mathrm{F}(3 / 54)=290, \mathrm{p}<.01]$ and target shape $[\mathrm{F}(1 / 18=10,62, \mathrm{p}<.01]$ are highly significant. The interaction between target shape and eccentricity is not significant $[F(3 / 54)=1.62]$.

\section{DISCUSSION}

Using luminance threshold as the measure of detection, circular stimuli have been shown to require less energy for detection than bar-shape targets. In addition, the data indicate that this situation exists throughout the periphery. Since these forms were equivalent in area of visual angle, retinal eccentricity, and subjective appearance and task-defined response, differences cannot be attributed to amount of retinal exitation, region of retinal excitation, or knowledge of stimulus form. Only the pattern of excitation can account for these data.

The hypotheses of Graham et al. (1939) and Kincaid et al. (1960) are consistent with these results. In an unrelated problem, Wolf and Zigler (1963) obtained data from rectangular stimuli which may also support this position. Their data show that, as a rectangle approached a square in form, its threshold diminished. The argument that the contribution to threshold of any stimulus element decreases with distance from the center of that stimulus may. apply here as well. A very different possibility stems from the consideration that cortical cells responsive to contours receive their input from lower order units responsive to points along a continuum. The response to line stimuli, therefore, would have to be integrated from activity across a number of approximately aligned circular receptive fields. While feature detectors are not necessary to explain threshold data involving light detection alone, they may play an indirect role in experience which contributes to the effect.

While these data have implications for understanding sensory mechanisms at threshold, they may also be important in applied situations where individuals are required to monitor the visual field under marginal conditions, and stimulus sensitivity would be critical.

\section{REFERENCES}

Abernethy, C. N., \& Leibowitz, H. W. The effect of feedback on luminance thresholds for peripherally presented stimuli. Perception \& Psychophysics, 1971, 10, 172-174.

Appelle, S. Orientation of test-object affects visibility throughout visual field. American Journal of Optometry and Archives of American Academy of Optometry, 1973, 50, 940-943.

Blackwell, H. R. A literature survey of the effects of target size and shape upon visual detection. Journal of the Optical Society of America, $1957,47,114$.

Graham, C. H., Brown, R. H., \& Mote, F. A. The relation of size of stimulus and intensity in the human eye: I. Intensity thresholds for white lights. Journal of Experimental Psychology, 1939, 24, 555-573.

Helson, H., \& Fehrer, E. V. The role of form in perception. American Journal of Psychology, 1932, 44, 79-102.

Kincaid, W. M., Blackwell, H. R., \& Kristofferson, A. B. Neural formulation of the effects of target size and shape upon visual detection. Journal of the Optical Society of America, $1960,50,141-148$.

Leibowitz, H. W., \& Appelle, S. The effect of a central task on luminance thresholds for peripherally presented stimuli. Human Factors, 1969, 11, 387-392.

Wolf, E., \& Zigler, M. J. Effects of uniocular and binocular excitation of the peripheral retina with test fields of various shapes on binocular summation. Journal of the Optical Society of America, 1963, 53, 1199-1205.

Zusne, L. Visual perception of form. New York: Academic Press, 1970.

(Received for publication May 14, 1975.) 